\title{
Functional and Radiological Outcomes of Anterior Decompression and Posterior Stabilization via Posterior Transpedicular Approach in Thoracic and Thoracolumbar Pott's Disease: A Retrospective Study
}

\author{
Suryakant Singh ${ }^{1}$, Hitesh Dawar ${ }^{1}$, Kalidutta Das ${ }^{2}$, Bibhudendu Mohapatra $^{2}$, Somya Prasad ${ }^{3}$ \\ ${ }^{1}$ Department of Orthopaedics, Indian Spinal Injuries Centre, New Delhi, India \\ ${ }^{2}$ Department of Orthopedics and Spine Services, Indian Spinal Injuries Centre, New Delhi, India \\ ${ }^{3}$ Department of Occupational Therapy, Indian Spinal Injuries Centre, New Delhi, India
}

\section{Study Design: This is a retrospective study.}

Purpose: To determine the efficacy and safety of a posterior transpedicular approach with regard to functional and radiological outcomes in people with thoracic and thoracolumbar spinal tuberculosis.

Overview of Literature: Spinal tuberculosis can cause serious morbidity, including permanent neurological deficits and severe deformities. Medical treatment or a combination of medical and surgical strategies can control the disease in most patients, thereby decreasing morbidity incidence. A debate always existed regarding whether to achieve both decompression and stabilization via a combined anterior and posterior approach or a single posterior approach exists.

Methods: The study was conducted at the Indian Spinal injuries Centre and included all patients with thoracic and thoracolumbar Pott's disease who were operated via a Posterior transpedicular approach. Data regarding 60 patients were analyzed with respect to the average operation time, preoperative and postoperative, 6 months and final follow-up American Spinal Injury Association (ASIA) grading, bony fusion, implant loosening, implant failure, preoperative, postoperative, 6 months and final follow-up kyphotic angles, a loss of kyphotic correction, Oswestry disability index (ODI) score, and visual analog scale (VAS) score. Data were analyzed using either a paired $t$-test or a Wilcoxon Signed Rank test.

Results: The mean operation time was $260 \pm 30$ minutes. Fifty-five patients presented with evidence of successful bony fusion within a mean period of $6 \pm 1.5$ months. Preoperative dorsal and lumbar angles were significantly larger than postoperative angles, which were smaller than final follow-up angles. The mean kyphotic correction achieved was $12.11 \pm 14.8$, with a mean decrease of 5.97 and 19.1 in VAS and ODI scores, respectively.

Conclusions: Anterior decompression and posterior stabilization via a posterior transpedicular approach are safe and effective procedures, with less intraoperative surgical duration and significant improvements in clinical and functional status.

Keywords: Thoracolumbar spine; Pott's disease; Transpedicular approach; Posterior stabilization; Anterior decompression

Received Oct 22, 2016; Revised Dec 22, 2016; Accepted Jan 3, 2017

Corresponding author: Suryakant Singh

Department of Orthopaedics, Indian Spinal Injuries Centre, A-155, Sector 19 Noida, Uttar Pradesh-201 301, India

Tel: +91-9910493794, E-mail: coolaryansksingh@gmail.com 


\section{Introduction}

Spinal tuberculosis (TB) accounts for $2 \%$ of all cases of $\mathrm{TB}$, with up to $15 \%$ of cases being extrapulmonary $\mathrm{TB}$ and $50 \%$ being skeletal TB $[1,2]$. It commonly affects the lower thoracic and lumbar vertebrae, followed by the middle thoracic and cervical vertebrae. Pott disease maybe the first manifestation but is usually secondary to lung or abdominal involvement [2].

Spinal TB is a diagnostic dilemma with a huge treatment challenge and a potential to cause serious morbidity, permanent neurological deficits, and severe deformities [2]. Medical treatment or combined medical and surgical strategies can control the disease in most patients [3], with the key being early detection and timely and judicious surgical intervention. This intervention depends on the clinicoradiological compression of the spinal cord and nerve roots, patient's age, and anti-tubercular treatment (ATT) responsiveness [2].

Surgical management helps regain motor function and ameliorates disability. Currently, the selection of an optimal surgical approach for treating thoracolumbar TB remains controversial. The goals of the operation are adequate decompression, debridement, reinforcement of stability, and correction and prevention of any deformity $[4,5]$.

The anterior approach was traditionally preferred because it enabled direct access to the infected focus and provided easier access for debridement and reconstruction of defects. Unfortunately, concomitant osteoporosis associated with the infection prevented adequate fixation in the thoracic and lumbar region [4,5]. A combined anterior and posterior approach helped overcome stabilityrelated drawbacks of an anterior approach, although it had its own drawbacks [5]. Posterior or posterolateral approaches have gained popularity in the last decade. Via the posterior extrapleural approach, both the anterior and lateral columns can be accessed [5], thereby providing excellent exposure for circumferential spinal cord decompression and enabling extension to multiple levels above and below the pathology level [4], with less morbidity and short operation time, as well as reduced blood loss, compared with the combined anterior and posterior approach [5]. Moon et al. [6] concluded that posterior spinal stabilization and anterior interbody fusion were helpful for preventing the disease early, providing an early fusion, and correcting and preventing kyphosis progression.
There has been limited research regarding the incorporation of a posterior transpedicular approach for surgically treating thoracolumbar TB. This study aimed to evaluate the efficacy and safety of decompression and stabilization via the posterior transpedicular approach alone for patients with thoracic and thoracolumbar Pott's disease.

\section{Materials and Methods}

\section{Subject and study design}

This is a Retrospective Cohort Study that included 60 patients with Thoracic and Thoracolumbar Pott's disease. This study was conducted at the Indian Spinal Injuries Centre (ISIC), New Delhi from June 2014 to June 2015 and included all patients with thoracic and Thoracolumbar Pott's disease from the inpatient department who underwent a posterior transpedicular approach procedure from January 2009 to January 2014. Patients aged $>18$ years who had thoracic and Thoracolumbar Pott's disease were eligible for the study. The diagnosis was confirmed in all patients by histopathological examination. Patients were included if they had deteriorating neurology while undergoing a conservative treatment (grade 1,2, and 3 paraplegics), severe kyphosis with an active disease $\left(>30^{\circ}\right.$ in adolescents and $>45^{\circ}$ in adults), progression of kyphotic deformity grade 4 paraplegics (as per Kumar's classification), limited improvement even with ATT for 4-6 weeks (grade 1,2, and 3 paraplegics), spinal instability on X-ray, and a spine with a risk sign of $\geq 2 / 4$.

Patients were excluded if the histology was consistent with another diagnosis, if they had cervical or sacral TB, if they underwent other surgical approaches, and if they had severe comorbidities and were deemed unfit for operation. The study was approved by the Institutional Review Board of ISIC Hospital and the Clinical Trials Registry of India (Trial no. CTRI/2016/09/007237). The demographic and clinical characteristics of the patients are shown in Table 1.

\section{Clinical evaluation}

The following outcome measures were evaluated at baseline, postoperatively, and at 6 and 12 months using a visual analog scale (VAS) for pain (Score of 0-10), Oswestry disability index (ODI) for low back pain (Score of 0-50), 
Table 1. Demographic and clinical characteristics of the patients included in the study

\begin{tabular}{|c|c|}
\hline Factor & Number $(\%)$ \\
\hline \multicolumn{2}{|l|}{ Age (yr) } \\
\hline$\leq 20$ & $3(5)$ \\
\hline $21-40$ & $13(21.66)$ \\
\hline $41-60$ & $31(51.66)$ \\
\hline $61-80$ & $12(20)$ \\
\hline$\geq 80$ & $1(1.66)$ \\
\hline \multicolumn{2}{|l|}{ Sex distribution } \\
\hline Male & $26(43.33)$ \\
\hline Female & $34(56.66)$ \\
\hline \multicolumn{2}{|l|}{ Level affected } \\
\hline Dorsal & 50 \\
\hline D1-D4 & $9(18)$ \\
\hline D5-D8 & $18(36)$ \\
\hline D9-D12 & $19(38)$ \\
\hline Multi-segment with skip lesions & $4(8)$ \\
\hline Dorsolumbar & $4(6.67)$ \\
\hline Lumbar & 6 \\
\hline $\mathrm{L} 1-\mathrm{L} 2$ & $2(33.33)$ \\
\hline $\mathrm{L} 2-\mathrm{L} 3$ & $1(16.66)$ \\
\hline L3- L4 & $1(16.66)$ \\
\hline L4-L5 & 2 (33.33) \\
\hline \multicolumn{2}{|l|}{ Preoperative ATT intake } \\
\hline Yes & 49 (81.66) \\
\hline No & $11(18.33)$ \\
\hline \multicolumn{2}{|l|}{ Histopathological evaluation } \\
\hline Done & $40(66.7)$ \\
\hline Not done & $20(33.3)$ \\
\hline
\end{tabular}

ATT, anti-tubercular treatment.

preoperative and postoperative X-ray; Cobb's angle for the kyphotic angle and loss of kyphotic correction; and the American Spinal Injury Association (ASIA) Scale for estimating the neurological status.

Besides these outcome measures, average operation time, bony fusion, implant loosening, and implant failure were evaluated.

\section{Statistical analysis}

A paired $t$-test or Wilcoxon signed rank test (in case of nonparametric data) was performed for comparing angles measured preoperative and postoperative and at the final follow-up, VAS scores, and ODI scores. Data were corrected using Bonferroni correction for multiple comparisons. The statistical significance level was set at $p<0.025$.

\section{Results}

From June 2014 to June 2015, 560 patients were identified as potential participants for the study, with 245 diagnosed as having thoracic and thoracolumbar TB and 85 undergoing the operation via the posterior transpedicular approach. Data of 60 patients (11\%) were used in this study.

The mean age was 49.7 years (range, $18-81$ years). Of the 60 patients included in the study, 34 were females and 26 were males. The upper thoracic spine D1-D4 was involved in nine patients, the middle thoracic spine D5D8 in 17, the lower thoracic spine D9-D12 in 19, and the dorsolumbar and lumbar involvement in 10. Five patients had multiple level involvement, from the upper thoracic spine to the dorsolumbar spine with skip lesions inbetween. Baseline characteristics are presented in Table 1.

\section{Primary endpoints: safety and efficacy}

Patients were examined for at least 12 months, with an average of 16.81 months (range, 12-40 months). The average preoperative erythrocyte sedimentation rate (ESR) and Creactive protein values were $45 \pm 15 \mathrm{~mm} / \mathrm{hr}$ and $29 \pm 15 \mathrm{mg} /$ $\mathrm{L}$, respectively, which decreased over 1-2 months after the operation and returned to normal at 3-6 months after the operation. No recurrence of spinal TB was observed at the final follow-up. The mean operation time was $260 \pm 30$ minutes, ranging from 180 to 540 minutes, with the maximum time recorded for patients who presented with either contiguous or continuous multiple level involvements.

The mean hospital stay for postoperative rehabilitation was 15 days, with the maximum being 80 days and the minimum being 6 days. The abbreviated injury score- $\mathrm{A}$ (AIS-A) patients required a prolonged hospital stay for rigorous physiotherapy to have an independent lifestyle, as per our institutional protocols.

\section{Secondary endpoints: clinically relevant outcomes}

Table 2 summarizes the changes in clinical outcomes at 
Table 2. Changes in clinical outcomes preoperatively (baseline), postoperatively, 6 months and at 12 months after the surgery

\begin{tabular}{|c|c|c|c|c|}
\hline Outcome & Preoperative & Postoperative & $6 \mathrm{mo}$ & 12 mo/final follow-up \\
\hline \multicolumn{5}{|c|}{ Neurological status } \\
\hline AIS-A & 3 & 3 & 4 & 4 \\
\hline AIS-B & 10 & 4 & 0 & 0 \\
\hline AIS-C & 20 & 11 & 2 & 2 \\
\hline AIS-D & 17 & 31 & 11 & 10 \\
\hline AIS-E & 10 & 11 & 43 & 44 \\
\hline \multicolumn{5}{|c|}{ Kyphotic angle } \\
\hline$<0^{\circ}$ & 4 & 5 & 5 & 5 \\
\hline $0^{\circ}-10^{\circ}$ & 4 & 25 & 16 & 12 \\
\hline $11^{\circ}-20^{\circ}$ & 10 & 27 & 33 & 37 \\
\hline $21^{\circ}-30^{\circ}$ & 35 & 3 & 4 & 4 \\
\hline $31^{\circ}-40^{\circ}$ & 7 & 0 & 2 & 2 \\
\hline \multicolumn{5}{|l|}{ VAS score } \\
\hline$<4$ & 0 & 59 & 60 & 60 \\
\hline $4-8$ & 38 & 1 & 0 & 0 \\
\hline$>8$ & 22 & 0 & 0 & 0 \\
\hline \multicolumn{5}{|c|}{ Oswestry disability index } \\
\hline$<10$ & 0 & 14 & 43 & 57 \\
\hline $11-20$ & 2 & 38 & 16 & 0 \\
\hline $21-30$ & 22 & 7 & 1 & 3 \\
\hline $31-40$ & 30 & - & 0 & 0 \\
\hline $41-50$ & 6 & 0 & 0 & 0 \\
\hline
\end{tabular}

AIS, abbreviated injury score; VAS, visual analog scale.

baseline, postoperative, and at 6 and 12 months after the operation. Fifty-five patients had a successful bony fusion within a mean of $6 \pm 1.5$ months, whereas five patients had either late fusion or nonfusion because of secondary infections. Of these five patients, one required a revision operation because of a surgical site infection. The other four were strictly followed up, but no active surgical intervention was performed (Fig. 1).

The mean preoperative kyphotic angle, as measured by Cobb's method, was $19.845^{\circ}$ (range from $-39.5^{\circ}$ to $37.76^{\circ}$ ) and the mean of immediate postoperative angle was $7.74^{\circ}$ (range from $-52.45^{\circ}$ to $24.29^{\circ}$ ). The mean correction achieved was $10.28^{\circ}$. The mean kyphotic angles measured at 6 and 12 months were $9.16^{\circ}$ and $9.67^{\circ}$, respectively. At the final follow-up, the mean kyphotic angle was $9.98^{\circ}$ and the mean loss of correction was $2.40^{\circ}$. These results are compatible with those of other studies (Fig. 2).

The maximum preoperative VAS score was 10 , with the minimum score being 6 and the mean score being 8.15. The maximum postoperative VAS score was 5 , with the minimum being 1 and the mean value being 2.18. The VAS score at the final follow-up was 3 , with the minimum being 0 and the mean value being 0.83 (Fig. 3). The mean preoperative ODI score was 32.52 , with the maximum score being 47 and the minimum score being 15 . The mean postoperative ODI score was 13.42 , with the maximum score being 30 and the minimum score being 5 . The corresponding data at the final follow-up were $3.96,0$, and 25 at 3, 6, and 12 months, respectively.

Thirty-five of 60 patients were categorized as AIS-C and AIS-D. Three patients were AIS-A, AIS-B, and AIS-E had equivalent number of patients, which are 11 in each category. Except four patients, the neurological status of all other patients improved postoperatively. At the final follow-up, 43 patients returned to their previous neurological level of AIS-E, and 11 patients regained their 

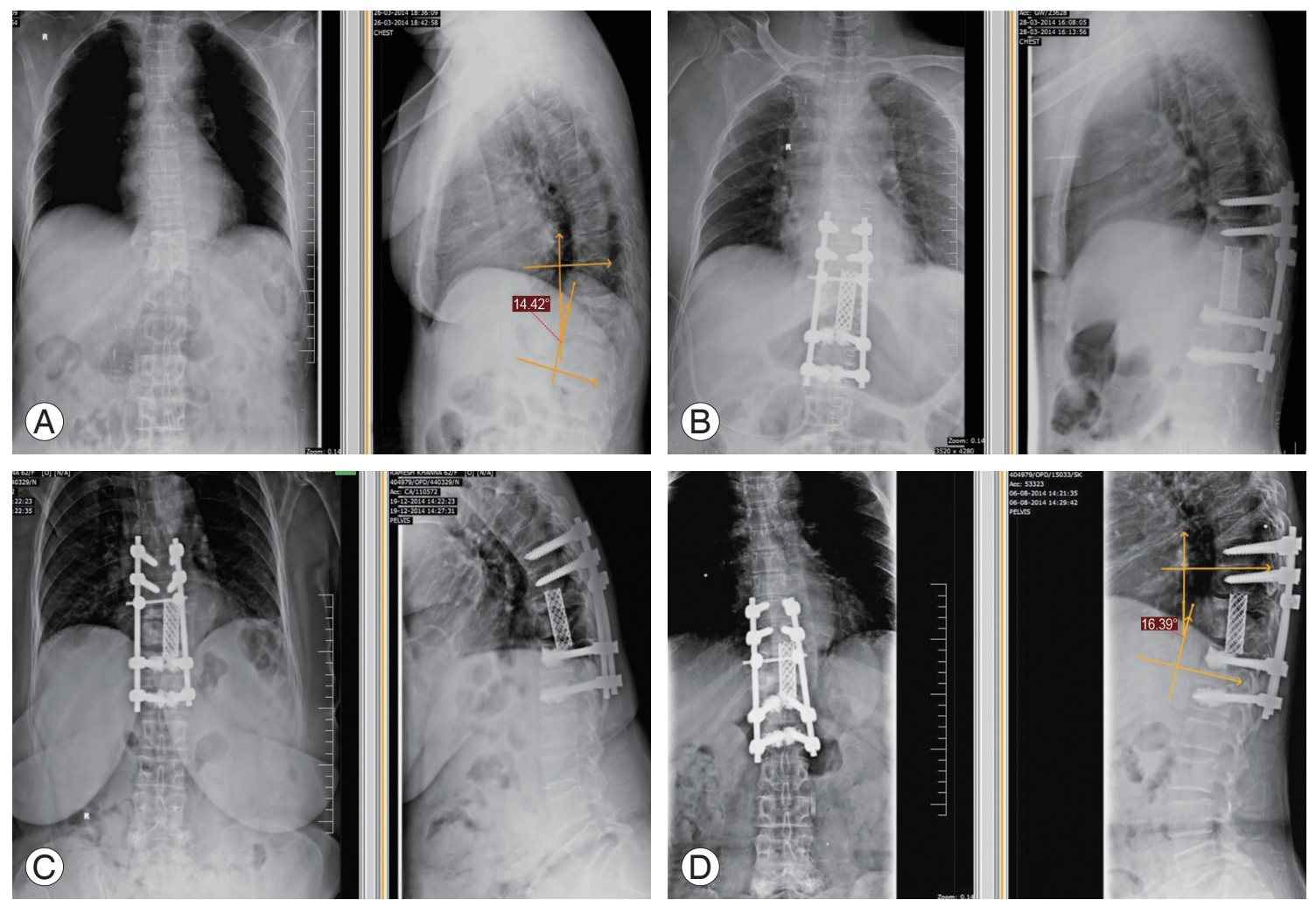

Fig. 1. Radiographs of a patient. Left, anteroposterior; right, lateral view. (A) Preoperative X-rays of a patient. (B) Immediate postoperative X-ray. (C) Six months postoperative X-ray. (D) Follow-up X-ray at 12 months.

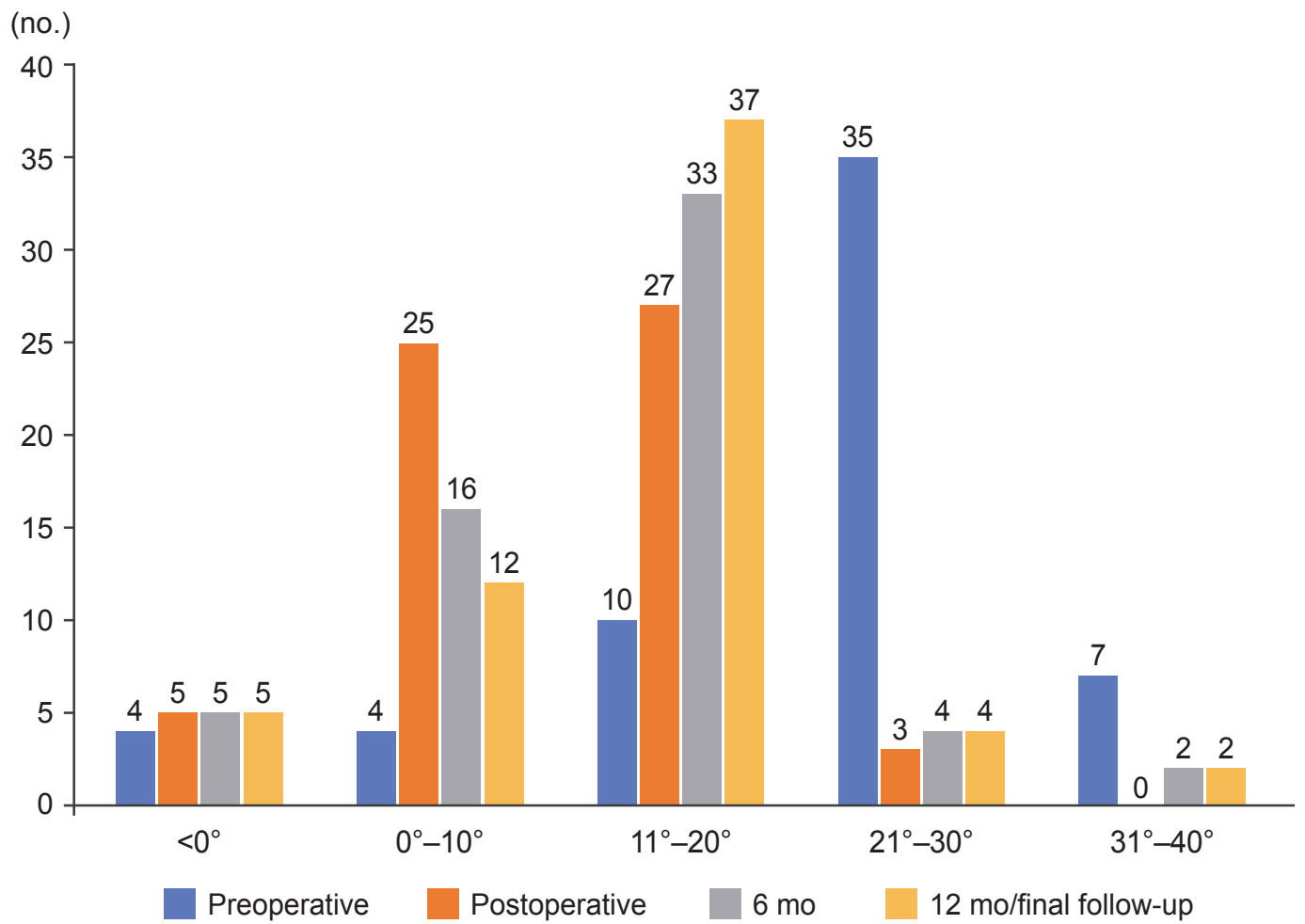

Fig. 2. Distribution of the patients according to the Kyphotic angles. 
neurological status and improved in the AIS-A scale from AIS-B to AIS-D. Three patients with AIS-A did not neurologically improve after the operation even at final follow-up (Fig. 4).

One patient was AIS-D before the operation but became AIS-A postoperatively owing to a perioperative complication of nerve root/cord damage and became AIS-A at the final follow-up. Another patient required an additional operation for the recurrence of symptoms and the development of an abscess. Two other patients developed postoperative infections (surgical site, which required debridement and secondary closure) and chest complication that prolonged their hospital stay and rehabilitation.

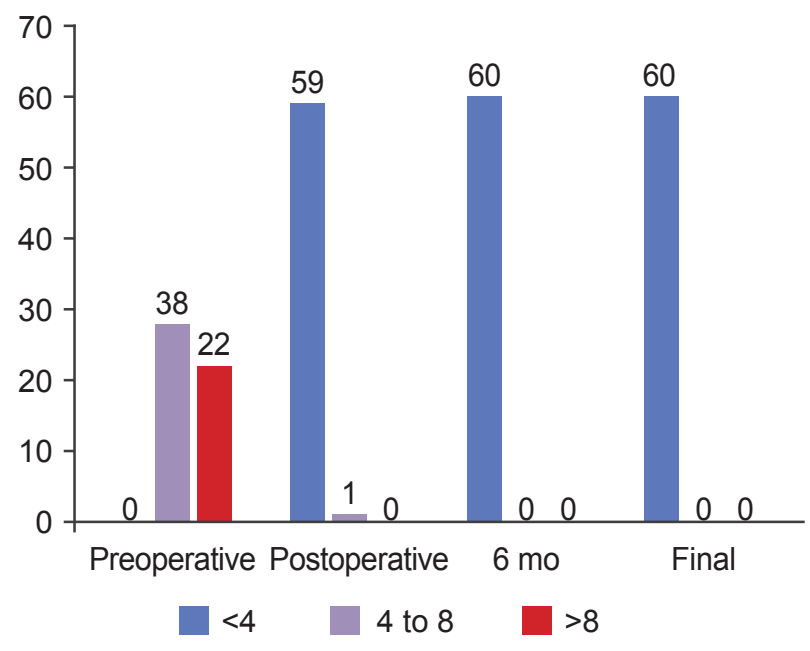

Fig 3. Distribution of the patients based upon the visual analog scale score.

\section{Discussion}

Pott's disease is the most common granulomatous bacterial infection of the spine and the most common bone TB $[4,7]$. The goals of the treatment include eradication of the infection [8], prevention and/or treatment of the neurological deficits, correction of the spinal deformities, achievement of a normal spinal contour, and achievement of an unrestricted ability to function in activities of daily life $[7,9,10]$. Excellent results in terms of clinical and radiological prognoses can be achieved with the treatment of spinal TB, provided there is an early diagnosis [4].

Several surgical options have been reported in the literature for spinal TB, such as anterior or posterior operation alone or a combination of both $[7,11]$. This study aimed to investigate the effects of the posterior transpedicular approach on the functional and radiological outcomes in people with thoracic and thoracolumbar Pott's disease. The study findings showed that the posterior transpedicular approach produced a clinically relevant improvement in the functionality of the patients.

In our study, patients underwent anterior decompression and posterior stabilization through the posterior transpedicular approach, leading to rapid recovery and early mobilization. Statistically significant improvements were observed with respect to the ODI, VAS, and ASIA scores and Cobb's angle, indicating recovery and improvement in the functional ability of the patients.

In this study, we retrospectively evaluated the functional and radiological outcomes of the posterior transpedicular

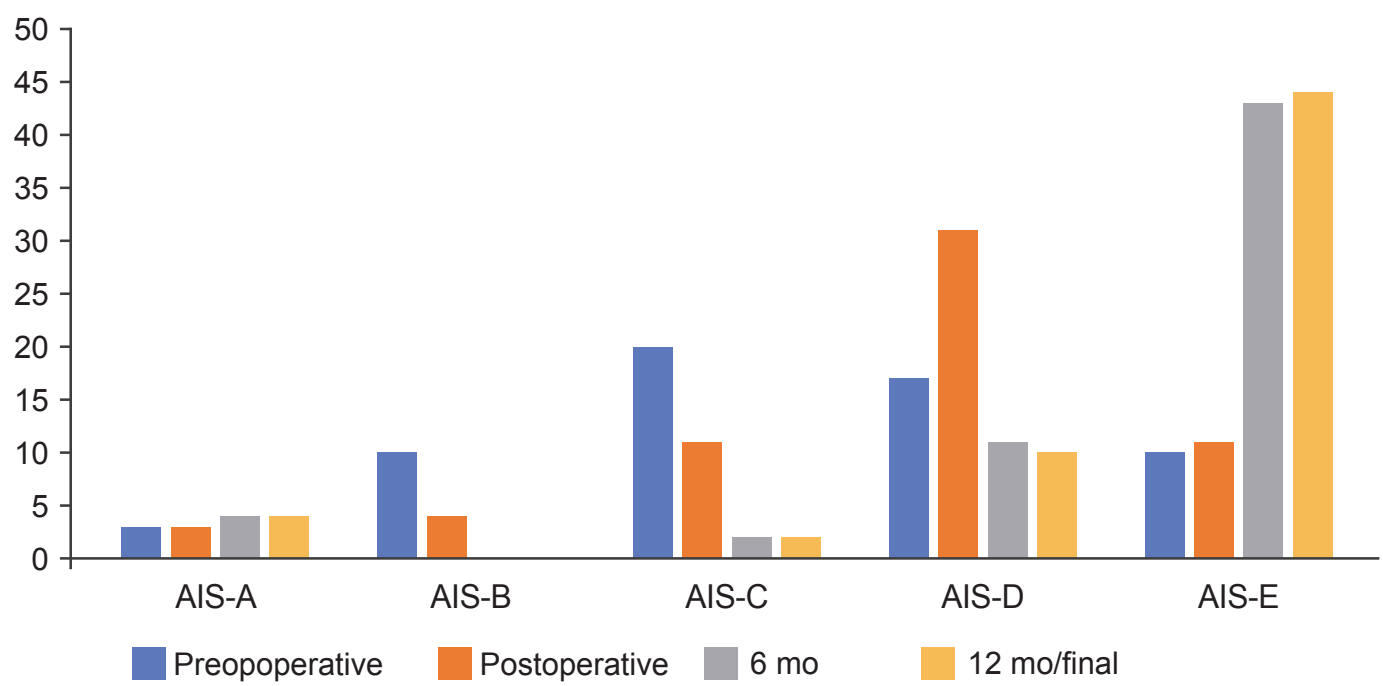

Fig. 4. Neurological status of the patients before and after the surgery (ASI-A grade). AIS, abbreviated injury score. 
approach only in 60 patients, of which $43 \%$ were males and $57 \%$ were females; these were similar to the figures in the studies by Sundararaj et al. [12] and Jain et al. [13]. The age of the patients ranged from 18 to 81 years. Schmorl et al. [14] reported that Pott's disease occurred in the first and second decade of life in $50 \%$ and $25 \%$ of patients, respectively. In our study, most patients were in the fourth to sixth decade of life. In a study by Jain et al. [13], the age distribution ranged from 2 to 57 years. Therefore, it can be concluded that patients of all ages are susceptible to spinal TB. Thus, a differential diagnosis should be considered in individuals who present with either back pain alone or back pain with a neurological deficit.

The average number of vertebra involved was 2.23 , with most patients displaying an involvement of two adjacent vertebrae, signifying that paradiscal lesions were the most common type of spinal TB [15]. Pott's disease requires surgical treatment when there is a loss of sagittal alignment of the spine, spread of an abscess in paraspinal tissues and the spinal canal, compression of the spinal cord or nerve root, and failed chemotherapy [16]. The most common indication for operation in our series was a progressive neurological deficit, despite ATT use, which was observed in almost 45 patients, with 32 having both neurological deficits and pain.

Surgical management depends on the following two principles: debridement with spinal cord decompression and stabilization [16]. The anterior approach described by AR Hodgson [5,16] in 1960 has been considered the gold standard for surgically treating Pott's disease because it enables a direct access to the vertebral body for debridement and an optimal visualization of lesions $[11,16]$. It also provides a large area for fusion [11]. However, the anterior approach has many limitations such as the requirement for long periods of immobilization, a progression of kyphosis, and a graft failure [11]; it also has a high morbidity rate [11]. Therefore, the posterior transpedicular approach, described by Hibbs and Albee, is an effective method to stabilize the thoracolumbar spine and promote healing [16]. This approach has been reported for preventing graft complications and kyphosis progression [5].

The posterior transpedicular approach also reduces the total operation time and morbidity, thereby enabling early mobilization [4], and offers excellent exposure to the spinal canal for decompression, rendering good clinical results $[4,11]$. Our findings are consistent with those reported by Sahoo et al. [17], who retrospectively evaluated the results of posterior decompression and transpedicular screw fixation in patients with thoracolumbar spinal TB and neurological deficits. They used posterior decompression and transpedicular screw fixation in a single stage with ATT and observed improved kyphosis. Bony fusion was achieved in $55 \%$ of patients, and neurological recovery occurred in $94.4 \%$ of patients. They further reported that all patients were relieved of pain, with the final VAS score ranging from 0 to 2 [17].

$\mathrm{Xu}$ et al. [18] found that after an anterior radical debridement with a bone graft fusion and a posterior pedicle screw-rod system fixation, the Cobb's angle was $9.8^{\circ} \pm 3.3^{\circ}$ postoperatively. The kyphotic deformity was also corrected at an angle of $17.2^{\circ}$ in the thoracolumbar region. Their study further concluded that the procedure was effective for bony fusion, correcting spinal kyphosis, and reconstructing spinal stability in patients with thoracolumbar spinal TB [18].

Lee et al. reported that transpedicular instrumentation provides excellent prevention of kyphotic progression in patients with limited spinal bone destruction. Their study and another one by Broner et al. also stated that the immobilization achieved using posterior instrumentation might be useful for suppressing infection and providing a stable environment to prevent TB recurrence [9]. In our study, the posterior transpedicular approach prevented infection and promoted healing, which was demonstrated by a significant decrease in ESR values at 2-4 months postoperatively, with no disease recurrence.

Thus, it can be concluded that conservative chemotherapy should be considered as the mainstay of treatment in all early and moderately advanced spinal TB cases, whereas operation is the mainstay of treatment along with chemotherapy for advanced cases with neurological deficits and impending/worsening kyphosis. Long-term neurological improvement is achieved only via the posterior transpedicular approach [5]. Many studies have reported satisfactory results and several advantages using the posterior transpedicular approach. One of the advantages of the approach is that it provides good fixation via the posterior elements because the disease pathology is anterior [5]. The posterior transpedicular approach also provides excellent exposure for a good spinal cord decompression and enables the instrumentation to be extended to multiple levels above and below the pathology level $[4,11]$. Moreover, the approach improves the correction of sagittal alignment of the spine [5] and addresses all our goals 
for the management of spinal TB.

The posterior transpedicular approach provides better functional outcomes and kyphosis correction compared with the anterior approach [5]. Our study demonstrated the clinical efficacy and safety of the posterior transpedicular approach in patients with Pott's disease, focusing on neurological improvement, rate of infection after the operation, operation time, and hospitalization stay. However, our study has some limitations. First, this was a retrospective study with a small sample size of 60 patients. Second, follow-up was performed for a minimum of 12 months, which is a very short follow-up period. Third, the amount of blood loss was not calculated and radiographs were used to assess fusion instead of computed tomography.

Despite these limitations, this study shows that the posterior transpedicular approach is a safe surgical procedure for thoracic and thoracolumbar Pott's disease, resulting in a short operation time and low postoperative morbidity. The results indicate that a posterior transpedicular approach contributes to improved functional status and significantly improves neurological, radiological, pain, and functional scores, as well as fusion rates.

\section{Conclusions}

In summary, this study constitutes the initial step toward the understanding of potential benefits of posterior transpedicular approach. However, for further validation, more Randomized Clinical trials with a larger sample size and a longer follow-up period considering other perioperative factors in addition to comparing the results of such trials with other approaches to finally ascertain the effectiveness of the posterior transpedicular approach are required.

\section{Conflict of Interest}

No potential conflict of interest relevant to this article was reported.

\section{Acknowledgments}

The authors gratefully acknowledge the assistance of Dr. Deepak Raina for his conceptual and methodologic contributions and constructive criticism.

\section{References}

1. Tuli SM. Severe kyphotic deformity in tuberculosis of the spine. Int Orthop 1995;19:327-31.

2. Ansari S, Amanullah MF, Ahmad K, Rauniyar RK. Pott's spine: diagnostic imaging modalities and technology advancements. N Am J Med Sci 2013;5:40411.

3. Chauhan A, Gupta BB. Letters to editor: spinal tuberculosis. J Indian Acad Clin Med 2007;8:110-4.

4. Hafez AR, Fattouh M. One-stage posterior instrumentation and fusion for the treatment of tuberculous spondylodiscitis of dorsal and lumbar spine. J Am Sci 2012;8:85-90.

5. Garg B, Kandwal P, Nagaraja UB, Goswami A, Jayaswal A. Anterior versus posterior procedure for surgical treatment of thoracolumbar tuberculosis: a retrospective analysis. Indian J Orthop 2012;46:165-70.

6. Moon MS, Moon YW, Moon JL, Kim SS, Sun DH. Conservative treatment of tuberculosis of the lumbar and lumbosacral spine. Clin Orthop Relat Res 2002;(398):40-9.

7. Karaeminogullari O, Aydinli U, Ozerdemoglu R, Ozturk C. Tuberculosis of the lumbar spine: outcomes after combined treatment of two-drug therapy and surgery. Orthopedics 2007;30:55-9.

8. Hosseinpour-Feizzi H, Elmi A. Surgical treatment results of spinal tuberculosis with combined anterior and posterior approach. J Med Sci 2007;7:790-6.

9. Ma YZ, Cui X, Li HW, Chen X, Cai XJ, Bai YB. Outcomes of anterior and posterior instrumentation under different surgical procedures for treating thoracic and lumbar spinal tuberculosis in adults. Int Orthop 2012;36:299-305.

10. Pu X, Zhou Q, He Q, et al. A posterior versus anterior surgical approach in combination with debridement, interbody autografting and instrumentation for thoracic and lumbar tuberculosis. Int Orthop 2012;36: 307-13.

11. Zaveri G. The role of posterior surgery in spinal tuberculosis. ArgoSpine News J 2011;23:112-9.

12. Sundararaj GD, Behera S, Ravi V, Venkatesh K, Cherian VM, Lee V. Role of posterior stabilisation in the management of tuberculosis of the dorsal and lumbar spine. J Bone Joint Surg Br 2003;85:100-6.

13. Jain AK, Aggarwal A, Dhammi IK, Aggarwal PK, Singh S. Extrapleural anterolateral decompression in 
tuberculosis of the dorsal spine. J Bone Joint Surg Br 2004;86:1027-31.

14. Schmorl G, Goin LS, Junghanns H, Wilk SP. The human spine in health and disease: anatomicopathologic. New York: Grune \& Stratton; 1959.

15. Garg RK, Somvanshi DS. Spinal tuberculosis: a review. J Spinal Cord Med 2011;34:440-54.

16. Varatharajah S, Charles YP, Buy X, Walter A, Steib JP. Update on the surgical management of Pott's disease. Orthop Traumatol Surg Res 2014;100:229-35.
17. Sahoo MM, Mahapatra SK, Sethi GC, Dash SK. Posterior-only approach surgery for fixation and decompression of thoracolumbar spinal tuberculosis: a retrospective study. J Spinal Disord Tech 2012;25:E21723.

18. Xu YG, Yang YD, Liu SL. Effect of surgical treatment for thoracolumbar spinal tuberculosis by anterior radical debridement with bone graft fusion and posterior pedicle screw-rods system fixation. Zhongguo Gu Shang 2009;22:938-40. 